\title{
Модельные оценки квантовой емкости аморфных и эпитаксиальных графеноподобных соединений
}

\author{
(C) С.Ю. Давыдов \\ Физико-технический институт им. А.Ф. Иофрфе Российской академии наук, \\ 194021 Санкт-Петербург, Россия \\ E-mail: Sergei_Davydov@mail.ru
}

Поступила в Редакцию 26 августа 2020 г.

В окончательной редакции 30 сентября 2020 г.

Принята к публикации 5 октября 2020 г.

\begin{abstract}
Предложены модели плотностей состояний аморфных графена и бинарных графеноподобных соединений, с помощью которых выполнены оценки квантовой емкости этих структур. Показано, что аморфизация понижает величину квантовой емкости. Тот же эффект имеет место, когда графен или графеноподобное соединение взаимодействует с металлической подложкой, тогда как в случае полупроводниковой (диэлектрической) подложки имеет место увеличение квантовой емкости.
\end{abstract}

Ключевые слова: графен, графеноподобное соединение, металлическая и полупроводниковая подложки, модельная плотность состояний.

DOI: 10.21883/FTP.2021.02.50506.9513

\section{1. Введение}

Впервые вопрос о квантовой емкости (КЕ) $C_{Q}$ был поднят для двумерного электронного газа в работе [1] и приобрел заметную популярность в связи с появлением графеновой тематики [2-9]. Обсуждалась КЕ однолистного $[3,5-7,9]$ и двухлистного $[5,7]$ графенов, эпитаксиального графена на карбиде кремния [8], графеновой наноленты $[3,4,9]$ и одномерной углеродной структуры - карбина [9]. Более того, в недавно появившихся статьях [10-12] вопрос о КЕ графеновых структур обсуждается в связи с проблемой суперконденсаторов и хранения энергии [13-15]. С другой стороны, одним из следствий большого числа работ по функционализации графена для приборного применения стал поиск (в основном теоретический) графеноподобных соединений (GLC), среди которых несомненный интерес представляют двумерные (2D) аналоги классических трехмерных (3D) полупроводников $\mathrm{A}_{N} \mathrm{~B}_{8-N} \quad$ [16-18]. Оценки $C_{Q}$ для $2 \mathrm{D} \mathrm{A}_{N} \mathrm{~B}_{8-N}$ были впервые сделаны в работе [19].

В цитированных работах (за исключением [8]) речь шла о свободных (не связанных с подложкой) идеальных кристаллических структурах. Здесь мы предлагаем первые модельные оценки КЕ для свободных аморфных структур однолистного графена (SLG) и GLC. Целью работы является ответ на вопрос, как аморфизация влияет на КЕ этих структур. С той же целью мы рассмотрим эпитаксиальные структуры, сформированные на металле, полупроводнике и диэлектрике.

\section{2. Аморфные структуры}

\section{1. Графен}

В теоретических работах [20-23] было установлено, что присутствие в двумерной сетке графена конечной концентрации пентагонов и гептагонов наряду с гексагонами приводит к ненулевой плотности состояний в окрестности точки Дирака. На этом основании в [24] была предложена модель плотности состояний аморфного однолистного графена (ASLG) вида

$$
\rho_{\mathrm{ASLG}}(\omega)=D \cdot \begin{cases}\sqrt{\omega^{2}+w^{2}}, & 0 \leq|\omega| \leq t, \\ \frac{t R}{|\omega|}, & t<|\omega| \leq 3 t, \\ 0, & |\omega|>3 t,\end{cases}
$$

где $\omega-$ энергетическая переменная, $D=(c / t R)$ $\times\left(1+\left(c w^{2} / 2 t R\right) \ln [(t+R) / w]\right)^{-1}-$ нормировочный множитель, $c=2 /(1+2 \ln 3), R=\sqrt{t^{2}+w^{2}}, t-$ ин- $^{-}$ теграл перехода между ближайшими соседними (б.с.), $w$ - энергия, характеризующая аморфизацию, проявляющуюся в ненулевом значении $\rho_{\mathrm{AG}}(\omega)$ в точке Дирака, принятой за нуль энергии. Здесь мы игнорируем энергетическое расплывание потолка зоны проводимости, дна валентной зоны (что свойственно аморфным материалам [25]) и возможную асимметрию зон, наводимую аморфизацией [21]. При $w=0$ выражение (1) переходит в плотность состояний, использованную в работе [9] для оценки КЕ однослойного кристаллического графена (SLG). Приведенная плотность состояний $\rho_{\mathrm{ASLG}}^{*}(\omega)=\rho_{\mathrm{ASLG}}(\omega) t$ для различных значений $w$ представлена на рис. 1 .

Воспользовавшись общим выражением для КЕ, приведенным в [2], получим:

$$
C_{Q}^{\mathrm{ASLG}}=\left(e^{2} / 4 T S\right) \int_{0}^{\infty} \rho_{\mathrm{ASLG}}(\omega) A\left(\omega, V^{*}\right) d \omega,
$$

$$
A\left(\omega, V^{*}\right)=\operatorname{sch}^{2}\left[\left(\omega-V^{*}\right) / 2 T\right]+\operatorname{sch}^{2}\left[\left(\omega+V^{*}\right) / 2 T\right],
$$

где $V^{*}=e V_{e l}-$ сдвиг электронных состояний, вызванный внешним электростатическом потенциалом $V_{e l}$, 


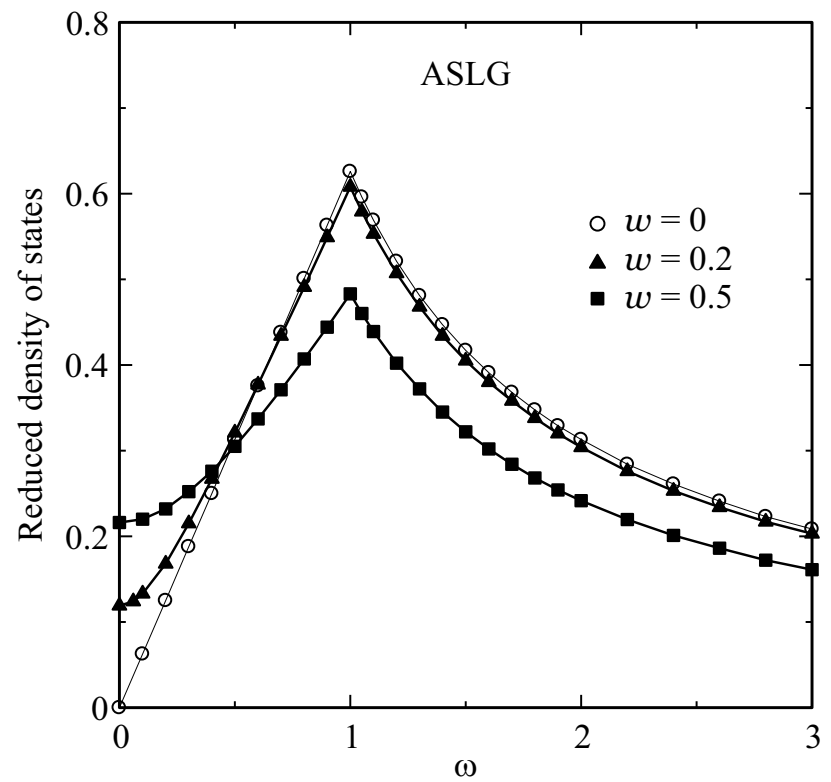

Рис. 1. Приведенная плотность состояний аморфного однолистного графена для $\rho_{\mathrm{ASLG}}^{*}(\omega)=\rho_{\mathrm{ASLG}}(\omega) t$ для $w=0,0.2$ и 0.5 . Все энергетические параметры измеряются в единицах $t$.

$e$ - величина заряда электрона, $T-$ температура в энергетических единицах, $S=3 \sqrt{3} a^{2} / 4$ - площадь, приходящаяся на один атом графена, $a$ - расстояние между б. с.; при $V^{*}=0$ химический потенциал совпадает с точкой Дирака. Перепишем (3) в виде

$$
\begin{gathered}
C_{Q}^{\mathrm{ASLG}}=C_{Q 1}^{\mathrm{ASLG}}+C_{Q 2}^{\mathrm{ASLG}}, \quad C_{Q 1,2}^{\mathrm{ASLG}}=\left(e^{2} D T / S\right) \bar{I}_{1,2} \\
\bar{I}_{1,2}=\bar{I}_{1,2}^{-}+\bar{I}_{1,2}^{+} \\
\bar{I}_{1}^{ \pm}=\int_{0}^{\tau} \sqrt{x^{2}+\bar{w}^{2}} \operatorname{sch}\left(x \pm v^{*}\right) d x \\
\bar{I}_{2}^{ \pm}=\tau r \int_{\tau}^{3 \tau} x^{-1} \operatorname{sch}\left(x \pm v^{*}\right) d x
\end{gathered}
$$

$x=\omega / 2 T, \quad \tau=t / 2 T, \quad \bar{w}=w / 2 T, \quad v^{*}=V^{*} / 2 T, \quad r=$ $=\sqrt{\tau^{2}+\bar{w}^{2}}$. В дальнейшем при комнатной температуре будем считать, что $\tau \gg 1$ и $\bar{w} \gg 1$.

Введем разность $\Delta C_{Q}^{\mathrm{ASLG}}=C_{Q 1}^{\mathrm{ASLG}}-C_{Q 2}^{\mathrm{SLG}}$, где, согласно [9], для кристаллического графена $C_{Q}^{\mathrm{SLG}}=$ $=C_{Q 1}^{\mathrm{SLG}}+C_{Q 2}^{\mathrm{SLG}}, \quad C_{Q 1,2}^{\mathrm{SLG}}=\left(e^{2} T / S\right) I_{1,2}, \quad I_{1,2}=I_{1,2}^{-}+I_{1,2}^{+}$, $I_{1}^{ \pm}=\int_{0}^{\tau} x \operatorname{sch}\left(x \pm v^{*}\right) d x, I_{2}^{ \pm}=\tau^{2} \int_{\tau}^{3 \tau} x^{-1} \operatorname{sch}\left(x \pm v^{*}\right) d x$. Пренебрежем интегралами $\bar{I}_{2}, I_{2}$ по сравнению с $\bar{I}_{1}, I_{1}$, как это делалось в [9] при вычислении KE SLG. Тогда получаем

$$
\Delta C_{Q}^{\mathrm{ASLG}} \approx\left(c e^{2} T / S t^{2}\right) F_{\mathrm{ASLG}}, \quad F_{\mathrm{ASLG}}=\left(t^{2} D / c\right) \bar{I}_{1}-I_{1} .
$$

В низшем порядке по параметру $w / t$ имеем $D \approx\left(c / t^{2}\right)\left[1-\left(c w^{2} / 2 t^{2}\right) \ln (2 t / w)\right]$, откуда получим

$$
F_{\mathrm{ASLG}} \approx\left(\bar{I}_{1}-I_{1}\right)-\left(c w^{2} / 2 t^{2}\right) \ln (2 t / w) I_{1},
$$

где $I_{1}=B\left(\tau, v^{*}\right)$ и функция $B\left(\tau, v^{*}\right)$ приведена в Приложении. Для первого слагаемого в (6) оценка дает $\bar{I}_{1}-I_{1} \approx \bar{w} C\left(\bar{w}, v^{*}\right)$, где функция $C\left(\bar{w}, v^{*}\right)$ приведена в Приложении. Если $v^{*} \ll \bar{w}$, получаем $\bar{I}_{1}-I_{1} \approx-4 \bar{w} \exp (-2 \bar{w}) \operatorname{ch}\left(2 v^{*}\right)$, если же $v^{*} \gg \bar{w} \gg 1$, имеем $\bar{I}_{1}-I_{1} \approx 4 \bar{w} \exp \left(-2 v^{*}\right) \operatorname{sh}(2 \bar{w})$. В обоих случаях имеем экспоненциально малую величину. Так как при $v^{*} \ll \tau$ интеграл $I_{1} \approx \ln \left\{2\left[1+\operatorname{ch}\left(2 v^{*}\right)\right]\right\}$ (см. Приложение), находим

$$
F_{\mathrm{ASLG}} \approx-\left(c w^{2} / 2 t^{2}\right) \ln (2 t / w) I_{1} .
$$

Таким образом, аморфизация графена приводит к уменьшению его КЕ. Отношение $\eta_{\mathrm{ASLG}}=\Delta C_{Q}^{\mathrm{ASLG}} / C_{Q}^{\mathrm{SLG}}$ $\approx-\left(c w^{2} / 2 t^{2}\right) \ln (2 t / w)$.

Рассмотрим дополнительно упрощенную модель аморфного графена, предложенную в работе [26], где плотность состояний ASLG задавалась в виде

$$
\rho_{\text {ASLG }}(\omega)=D^{\prime} \begin{cases}\sqrt{\omega^{2}+w^{2}}, & |\omega| \leq \xi \\ 0, & |\omega|>\xi\end{cases}
$$

$D^{\prime}=2\left(\xi \sqrt{\xi^{2}+w^{2}}+w^{2} \ln \left[\left(\xi+\sqrt{\xi^{2}+w^{2}}\right) / w\right]\right)^{-1}-$ нормировочный множитель, $\quad \xi=\sqrt{2 \pi \sqrt{3}} t-$ энергия обрезания [27]. Такая плотность состояний соответствует низкоэнергетическому приближению, в рамках которого дисперсия электронов считается линейной функцией [28]. Подставляя (8) в (2), имеем

$$
C_{Q}^{\mathrm{ASLG}}=\left(e^{2} D^{\prime} T / S\right) / \bar{I}, \quad \bar{I}=\int_{0}^{\bar{\xi}} \sqrt{x^{2}+\bar{w}^{2}} A\left(x, v^{*}\right) d x
$$

где $\bar{\xi}=\xi / 2 T$, откуда приходим к формуле (7) с заменой $t$ на $\xi$ и $c$ на 2. Отметим, что на рис. 1 плотности состояний (8) соответствует энергетический интервал $\omega / t$ от 0 до 1 .

\section{2. Графеноподобные соединения}

Перейдем теперь к оценкам КЕ для аморфных GLC (AGLC), плотность состояний которых примем равной

$\rho_{\mathrm{AGLC}}(\omega)=K \begin{cases}\Delta \exp [-(\Delta-|\omega|) / u], & |\omega|<\Delta, \\ |\omega|, & \Delta \leq|\omega| \leq \omega_{\max }, \\ 0, & |\omega|>\omega_{\max },\end{cases}$

где $K=\left(u \Delta[1-\exp (-\Delta / u)]+\xi^{2} / 2\right)^{-1}-$ нормировочный множитель, $u$ - энергетический параметр, характеризующий степень аморфизации, $\omega_{\max }=\sqrt{\xi^{2}+\Delta^{2}}$ и 


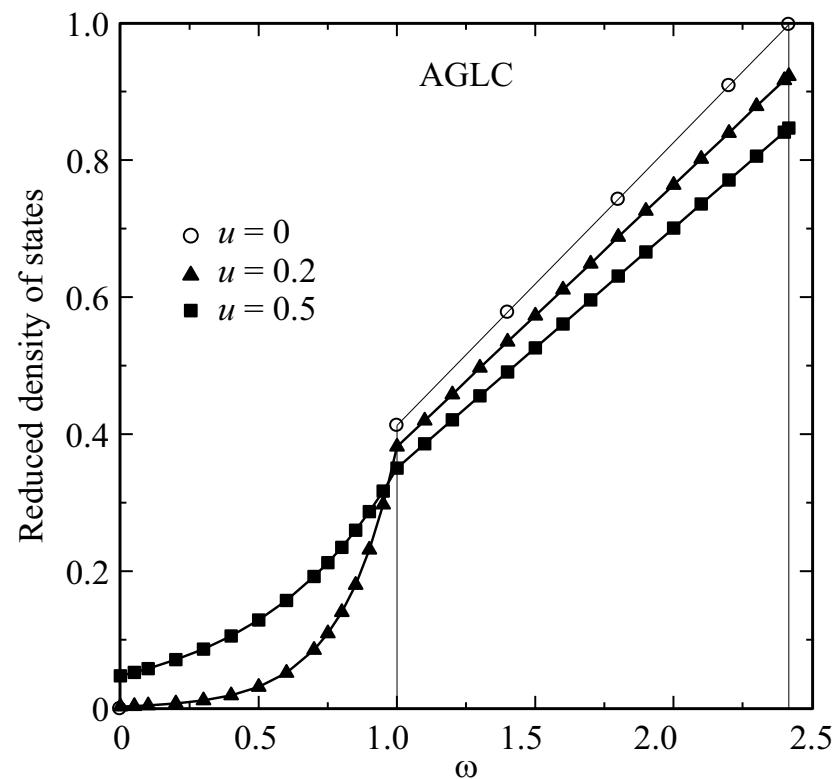

Рис. 2. Приведенная (безразмерная) плотность состояний аморфного графеноподобного соединения для $\rho_{\mathrm{AGLC}}^{*}(\omega)=$ $=\rho_{\mathrm{AGLC}}(\omega) \Delta, u=0,0.2$ и 0.5 . Все энергетические параметры измеряются в единицах $\Delta$.

$2 \Delta$ - энергия потолка зоны проводимости и ширина энергетической щели свободных GLC (за нуль энергии принят центр щели, с которым при $V^{*}=0$ перекрывается химический потенциал). Формула (10) соответствует низкоэнергетическому приближению и при $u=0$ переходит в соответствующее выражение для плотности состояний кристаллических GLC [29]. Здесь, как и выше, мы игнорируем вызванное аморфизацией расплывание периферийных участков плотности состояний. Приведенная плотность состояний аморфного графеноподобного соединения $\rho_{\mathrm{AGLC}}^{*}(\omega)=\rho_{\mathrm{AGLC}}(\omega) \Delta$ для различных значений $u$ представлена на рис. 2 .

Подставляя (10) в (2), найдем:

$$
\begin{gathered}
C_{Q}^{\mathrm{AGLC}}=\frac{e^{2} K T}{S}\left(J_{1}+J_{2}\right), \\
J_{1}=\delta \int_{0}^{\delta} \exp [-(\delta-x) \bar{u}] A\left(x, v^{*}\right) d x, \\
J_{2}=I\left(\delta, x_{\max } ; v^{*}\right),
\end{gathered}
$$

где $\quad \bar{u}=u / 2 T, \quad \delta=\Delta / 2 T, \quad x_{\max }=\omega_{\max } / 2 T \quad$ и функция $I\left(\delta, x_{\max } ; v^{*}\right)$ определена в Приложсении. Так как $\quad C_{Q}^{\mathrm{GLC}}=\left(2 e^{2} T / S \xi^{2}\right) I\left(\delta, x_{\max } ; v^{*}\right), \quad$ вводя $\Delta C_{Q}^{\mathrm{AGLC}}=C_{Q 1}^{\mathrm{GSLC}}-C_{Q 2}^{\mathrm{GLC}}$, получим

$$
\begin{gathered}
\Delta C_{Q}^{\mathrm{AGLC}}=\left(2 e^{2} T / S \xi^{2}\right) F_{\mathrm{AGLC}}, \\
F_{\mathrm{AGLC}}=K^{\prime} J_{1}+\left(K^{\prime}-1\right) J_{2} .
\end{gathered}
$$

При $u / \Delta \ll 1$ имеем $K^{\prime} \approx 1-2 u \Delta / \xi^{2}$, откуда получаем $\left(K^{\prime}-1\right) J_{2}=-\left(2 u \Delta / \xi^{2}\right) I\left(\delta, x_{\max } ; v^{*}\right)$. Учитывая, что в интеграл $J_{1}$ основной вклад дает интервал $(\delta-\bar{u}, \delta)$, имеем $K^{\prime} J_{1} \approx \delta\left[C\left(\delta, v^{*}\right)-C\left(\delta-\bar{u}, v^{*}\right)\right]$. При $v^{*} \ll \delta$ получаем $\quad K^{\prime} J_{1} \approx-4 \delta \exp (-2 \delta)[1-\exp (-2 \bar{u})] \operatorname{ch}\left(2 v^{*}\right)$, т. е. экспоненциально малую величину. Тогда $F_{\mathrm{AGLC}} \approx-2\left(u \Delta / \xi^{2}\right) I\left(\delta, x_{\mathrm{max}} ; v^{*}\right)$, так что при $v^{*} \ll \delta$ имеем

$$
F_{\mathrm{AGLC}} \approx-2\left(u \Delta / \xi^{2}\right) \ln \left\{2\left[1+\operatorname{ch}\left(2 v^{*}\right)\right]\right\}
$$

и $\eta_{\mathrm{AGLC}}=\Delta C_{Q}^{\mathrm{AGLC}} / C_{Q}^{\mathrm{AGLC}} \approx-2 u \Delta / \xi^{2}$.

Таким образом, аморфизация GLC приводит к уменьшению КЕ. Отметим, что выражения (7) и (13) с качественной точки зрения одинаковы. Эти формулы показывают, что увеличение плотности состояний в области низких энергий, имеющее место при аморфизации кристаллических SLG и GLC, играет меньшую роль, чем понижение плотности состояний при больших энергиях. Отметим также, что уменьшение КЕ пропорционально $w, u, V^{*}$. При этом $\Delta C_{Q}^{\mathrm{ASLG}}(w=0)=0 \quad$ и $\quad \Delta C_{Q}^{\mathrm{EGLC}}(u=0)=0, \quad$ но $\Delta C_{Q}^{\mathrm{ASLG}}\left(V^{*}=0\right) \neq 0$ и $\Delta C_{Q}^{\mathrm{AGLC}}\left(V^{*}=0\right) \neq 0$.

\section{3. Эпитаксиальные структуры}

Для эпитаксиальных структур характеристиками взаимодействия слоя с подложкой являются функция уширения $\Gamma(\omega)=\pi V^{2} \rho_{\text {sub }}(\omega)$ и функция сдвига $\Lambda(\omega)$, связанные соотношением

$$
\Lambda(\omega)=\frac{1}{\pi} P \int_{-\infty}^{\infty} \frac{\Gamma\left(\omega^{\prime}\right) d \omega^{\prime}}{\omega-\omega^{\prime}},
$$

где $V$ - матричный элемент взаимодействия слой-подложка, $\rho_{\mathrm{sub}}(\omega)-$ плотность состояний подложки, $P$ символ главного значения интеграла [27]. Плотность состояний EGLC в общем виде есть

$$
\begin{gathered}
\rho_{\mathrm{EGLC}}(\Omega)=\frac{2 \hat{I}(\Omega)}{\xi^{2}}, \\
\hat{I}(\Omega)=\frac{\Gamma(\omega)}{2 \pi} \ln \frac{\left|\xi^{4}+b^{\prime} \xi^{2}+c\right|}{c^{\prime}} \\
+\frac{\Omega}{\pi}\left(\operatorname{arctg} \frac{2 \xi^{2}+b^{\prime}}{4 \Gamma(\omega) \Omega}-\operatorname{arctg} \frac{b^{\prime}}{4 \Gamma(\omega) \Omega}\right),
\end{gathered}
$$

где $\Omega=\omega-\varepsilon_{\mathrm{D}}-\Lambda(\omega) \quad\left(\varepsilon_{\mathrm{D}}-\right.$ энергия точки Дирака), $\quad b^{\prime}=-2\left[\Omega^{2}-\Delta^{2}-\Gamma^{2}(\omega)\right], \quad c^{\prime}=\left(\Omega^{2}-\Delta^{2}\right)^{2}$ $+\Gamma^{2}(\omega)\left[\Gamma^{2}(\omega)+2 \Delta^{2}+2 \Omega^{2}\right] \quad[29]$. Положив $\Delta=0$, получим плотность состояний $\rho_{\mathrm{ESLG}}(\Omega)[29]$. В дальнейшем будем рассматривать только режим слабой связи наноструктур с подложками, когда характерное значение функции $\Gamma(\omega)$ мало по сравнению с энергией взаимодействия $t$ ближайших атомов в эпитаксиальном слое. Отметим, что в режиме сильной связи слой теряет свою специфику, приобретая черты изолированных адатомов. 


\section{1. Металлическая подложка}

Простейшей моделью плотности состояний металлической подложки $\rho_{m}(\omega)$ является модель Андерсона, предполагающая $\rho_{m}(\omega)=\mathrm{const}$, откуда следует, что $\Gamma(\omega)=\Gamma_{m}=$ const $\quad$ и $\Lambda_{m}(\omega)=0 \quad[30,31]$. Именно эта модель использовалась в $[29,32]$ для описания эпитаксиального однолистного графена (ESLG) и эпитаксиальных графеноподобных соединений (EGLC) соответственно. В такой модели режиму слабой связи отвечает неравенство $\Gamma_{m} \ll \xi$, где, как и выше, энергия обрезания $\xi=\sqrt{2 \pi \sqrt{3}} t$ возникает вследствие использованного низкоэнергетического приближения.

Функции $\quad I_{\mathrm{ESLG}}^{*}(\omega)=\hat{I}(\omega, \Delta=0) / t=\rho_{\mathrm{ESLG}}(\omega) \xi^{2} / 2 t$ и $I_{\mathrm{EGLC}}^{*}(\omega)=\hat{I}(\omega) / t=\rho_{\mathrm{EGLC}}(\omega) \xi^{2} / 2 t \quad$ представлены на рис. 1 для случая $\varepsilon_{\mathrm{D}}=0 \quad(\Omega=\omega)$. Там же для сравнения приведены плотности состояний свободных графена, $I_{\mathrm{SLG}}^{0}(\omega), \quad$ и графеноподобного соединения, $I_{\mathrm{GLC}}^{0}(\omega)$. Слабый положительный пик функции $I_{\text {ESLG }}^{*}(\omega)$ имеет место при $\omega=0$, когда $\hat{I}(0) \approx(2 \Gamma / \pi) \ln (\xi / \Gamma)$. Отрицательный пик соответствует $\omega=\xi, \quad$ когда $\hat{I}(\xi) \approx-(2 \Gamma / \pi) \ln (\xi / \Gamma)+(\xi / 2)$. Для функции $I_{\mathrm{EGLC}}^{*}(\omega)$ имеем положительный пик $\hat{I}(\Delta) \approx(2 \Gamma / \pi) \ln (\xi / \Gamma)+(\Delta / 2) \quad$ при $\omega=\Delta \quad$ и отрицательный пик $\hat{I}\left(\omega_{\max }\right) \approx-(2 \Gamma / \pi) \ln (\xi / \Gamma)+\left(\omega_{\max } / 2\right)$ при $\omega=\omega_{\max }=\sqrt{\Delta^{2}+\xi^{2}}$. Отметим, что в точках $\omega=\Delta$ и $\omega=\omega_{\max }$ значения функций $\Omega^{2}-\Delta^{2}$ и $\xi^{2}-\Omega^{2}+\Delta^{2}$ равны $\Gamma_{m}^{2}$ (см. точные выражения в $\left.[29,32]\right)$.

Сравнение плотностей состояний эпитаксиальных (рис. 3) и свободных аморфных структур (рис. 1 и 2)

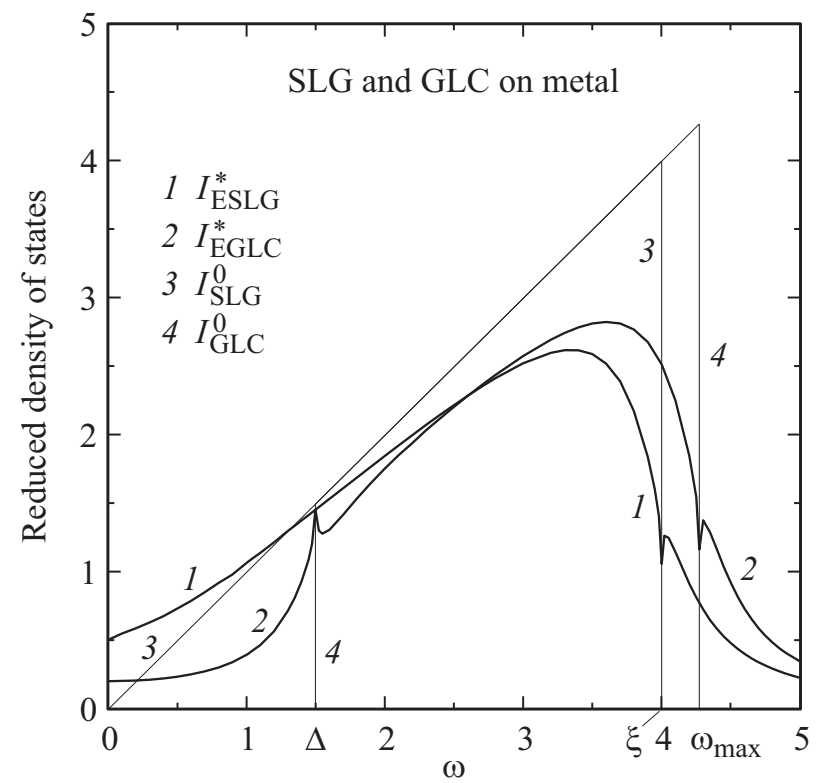

Рис. 3. Однолистный графен (SLG) и графеноподобное соединение (GLC) на металле: приведенные плотности состояний $I_{\mathrm{ESLG}}^{*}(\omega), I_{\mathrm{EGLC}}^{*}(\omega)$ и плотности состояний свободных графена $I_{\mathrm{SLG}}^{0}(\omega)$, GLC $I_{\mathrm{GLC}}^{0}(\omega)$ при $\varepsilon_{\mathrm{D}}=0$. Все энергетические величины измеряются в единицах $t: \Delta=1.5, \xi=4, \bar{\Gamma}_{m} / \pi=0.1$, $\omega_{\max }=\sqrt{\Delta^{2}+\xi^{2}}$. показывает их качественное сходство: имеет место рост $\rho_{\mathrm{EGLC}}(\omega), \rho_{\mathrm{ESLG}}(\omega)$ в области малых $\omega$ и спад в области больших $\omega$. Высокоэнергетические хвосты плотности состояний эпитаксиальных структур, отсутствующие в принятых нами моделях аморфных структур, практически не влияют на величину КЕ, так как вырезаются из соответствующих интегралов функцией $A\left(x, v^{*}\right)$. Поскольку здесь мы преследуем лишь качественные цели, отмеченное сходство позволяет оценить КЕ эпитаксиальных структур на металле, воспользовавшись результатами разд. 2 настоящей работы, и сделать вывод о том, что КЕ свободных структур превосходят КЕ эпитаксиальных структур на металле.

Отмеченное сходство плотностей состояний дает возможность связать введенные в разд. 2 эмпирические параметры $u$ и $w$ с характеристиками плотностей состояний ESLG и EGLC. Действительно, используя (10), при $\Gamma_{m} \ll \xi, w \ll \xi$ из равенства $\rho_{\mathrm{ASLG}}(0)=D^{\prime} w$ имеем

$$
w \approx\left(\Gamma_{m} / \pi\right) \ln \left(\xi / \Gamma_{m}\right) .
$$

Далее, приравнивая $\rho_{\mathrm{AGLC}}(0)=K \Delta \exp (-\Delta / u) \quad$ к $\rho_{\mathrm{EGLC}}(0)$, при $u \ll \Delta$ получим

$$
u=-\frac{\Delta}{\ln \left\{\left(\Gamma_{m} / 2 \pi \Delta\right) \ln \left[\left(\xi^{2}+\Delta^{2}\right) / \Delta^{2}\right]\right\}} .
$$

Отметим, что при $\Gamma_{m}=0$ параметры $w$ и $u$ обращаются в нуль.

\section{2. Полупроводниковая (диэлектрическая) подложка}

3.2.1. Модель подложки. Задача об эпитаксиальных графене и графеноподобном соединении на полупроводнике рассматривалась нами в ряде работ [32,33-35]. При этом для описания плотности состояний подложки использовались две модели $\rho_{\mathrm{sub}}(\omega)$ : модель ХалдейнаАндерсона $[30,33,34]$ и модель, соответствующая параболическому электронному спектру подложки [35]. В работе [35] в качестве подложки рассматривался произвольный полупроводник, в [33,34] - политипы карбида кремния; свободный графен описывался в низкоэнергетическом приближении. Вновь будем рассматривать режим слабой связи графен-подложка. Тогда легко понять, что если точка Дирака свободного графена $\varepsilon_{\mathrm{D}}$ лежит в глубине какой-либо из разрешенных зон, то мы имеем по сути „металлическую ситуацию“, рассмотренную в разд. 3.1. Поэтому интерес представляет случай, когда точка Дирака попадает в запрещенную зону подложки. Именно такая ситуация реализуется для гексагональных политипов $4 H-\mathrm{SiC}$ и $6 H-\mathrm{SiC}[34]$.

Воспользуемся моделью Халдейна-Андерсона, в которой плотность состояний полупроводника равна

$$
\rho_{s c}(\omega)= \begin{cases}\bar{\rho}_{s c}, & |\omega| \geq E_{g} / 2, \\ 0, & |\omega|<E_{g} / 2,\end{cases}
$$

где $E_{g}$ - ширина запрещенной зоны, центр которой принят за нуль энергии. Тогда функции полуширины и 
сдвига уровней графена равны соответственно

$$
\begin{gathered}
\Gamma_{s c}(\omega)=\pi V_{s c}^{2}(\omega) \rho_{s c}(\omega), \\
\Lambda_{s c}(\omega)=\left(\bar{\Gamma}_{s c} / \pi\right) \ln \left|\left(\omega-E_{g} / 2\right) /\left(\omega+E_{g} / 2\right)\right|,
\end{gathered}
$$

где $V_{s c}$ - матричный элемент взаимодействия графенподложка, и $\bar{\Gamma}_{s c}=\pi V_{s c}^{2} \bar{\rho}_{s c}$.

3.2.2. Графен. Приведенная плотность состояний эпитаксиального графена $I_{\mathrm{ESLG}}^{*}(\omega)=2 \hat{I}_{\mathrm{ESLG}} / E_{g}$ при $\varepsilon_{\mathrm{D}}$ представлена на рис. 4, там же для сравнения изображена плотность состояний свободного графена $I_{\mathrm{SLG}}^{0}(\omega)$. В области запрещенной зоны подложки $\quad I_{\mathrm{ESLG}}^{*}(\omega)=\Omega \quad$ и $I_{\mathrm{SLG}}^{0}(\omega)=\omega \quad$ [32]. Так как в области $0<\omega \leq E_{g} / 2$ функция $\Lambda_{s c}(\omega)<0$, имеем $\Omega>\omega$. При $\omega>E_{g} / 2$ почти вплоть до максимума плотности состояний $\left(I_{\mathrm{ESLG}}^{*}\right)_{\max }=I_{\mathrm{ESLG}}^{*}\left(\omega^{*}\right)$ имеем $I_{\mathrm{ESLG}}^{*}(\omega) \propto \Omega=\omega-\Lambda_{s c}(\omega)-$ функция, близкая к линейной при малых $\omega$. Узкий максимум $I_{\mathrm{ESLG}}^{*}(\omega)$ связан со ступенчатой плотностью состояний подложки в модели Халдейна-Андерсона. При $\omega>\omega^{*}$ наступает резкий спад $I_{\mathrm{ESLG}}^{*}(\omega)$. Имеем, таким образом, три характерные области изменения $I_{\text {ESLG }}^{*}(\omega)$, которые для простоты сведем к двум, представив КЕ в виде

$$
\begin{gathered}
C_{Q}^{\mathrm{ESLG}}=\sum_{t=1}^{2} C_{Q i}^{\mathrm{ESLG}}, \quad C_{Q i}^{\mathrm{ESLG}}=\left(2 e^{2} T L / S \xi^{2}\right) F_{\mathrm{ESLG}}^{i}, \\
F_{\mathrm{ESLG}}^{i}=\int_{\bar{a}_{i}}^{\bar{b}_{i}}\left[\hat{I}_{\mathrm{ESLG}}^{*}(x)\right]_{i} A\left(x, v^{*}\right) d x
\end{gathered}
$$

где $\hat{I}_{\mathrm{ESLG}}^{*}=\hat{I}_{\mathrm{ESLG}} / 2 T, \bar{a}_{1}=0, \bar{b}_{1}=\bar{a}_{2}=E_{g} / 4 T \equiv e_{g} / 2$, $\bar{b}_{2}=\infty, L-$ нормировочный множитель.

Рассмотрим энергетический интервал $\left(0, E_{g} / 2\right)$. Энергия точки Дирака графена относительно центра запрещенной зоны равна $\varepsilon_{\mathrm{D}}=\phi_{\mathrm{SLG}}-\chi-E_{g} / 2$, где $\phi_{\mathrm{SLG}}=4.79$ эB - работа выхода свободного графена $[36], \chi-$ электронное сродство полупроводника. Воспользовавшись приведенными в [34] значениями $E_{g}$ (3.23 и 3.00 эВ для политипов $4 H$ и $6 H$ соответственно) и $\chi(3.17$ и 3.45 эВ для политипов $4 H$ и $6 H)$, получим $\varepsilon_{\mathrm{D}}=0.005$ эВ для $4 H-\mathrm{SiC}$ и $\varepsilon_{\mathrm{D}}=-0.16$ эВ для $6 H-\mathrm{SiC}$, так что $2\left|\varepsilon_{\mathrm{D}}\right| / E_{g} \ll 1$. Для простоты положим $\varepsilon_{\mathrm{D}}=0$.

Далее, в области малых энергий в приближении слабой связи $2 \bar{\Gamma}_{s c} / E_{g} \ll 1$ имеем $\Omega \approx k_{1} \omega$, где $\hat{k}=1-\left(\partial \Lambda_{s c}(\omega) / \partial \omega\right)_{\omega=\omega^{*}}$. Учтем, что

$$
\partial \Lambda_{s c}(\omega) / \partial \omega= \pm 4 \bar{\Gamma}_{s c} E_{g} / \pi\left(4 \omega^{2}-E_{g}^{2}\right)
$$

где знак „плюс“ отвечает области $\omega>E_{g} / 2$, а знак „минус“ - области $\omega<E_{g} / 2$. Вычисляя $k_{1}$ при $\omega=0$, получим $k_{1}=1+4 \bar{\Gamma}_{s c} / \pi E_{g}$. Переходя к энергетическому интервалу $\left(E_{g} / 2, \infty\right)$, экстраполируем линейную зависимость $\Omega \approx k_{2} \omega$ на всю область $\left(E_{g} / 2, \xi\right)$, где $k_{2}=1-\bar{\Gamma}_{s c} E_{g} / \pi \xi^{2}$. При этом мы завышаем вклад плотности состояний $\rho_{\mathrm{ESLG}}(\omega)$ в КЕ в энергетическом интервале $\left(E_{g} / 2, \xi\right)$ и игнорируем вклад $\rho_{\mathrm{ESLG}}(\omega)$ в интервале

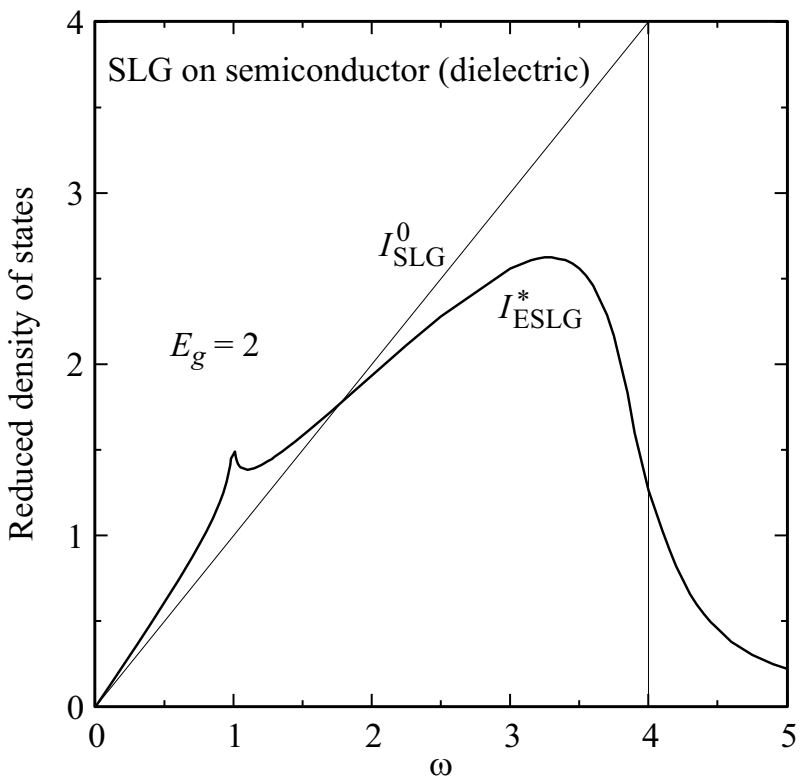

Рис. 4. Однолистный графен (SLG) на полупроводнике или диэлектрике: приведенные плотности состояний для эпитаксиальной $I_{\mathrm{ESLG}}^{*}(\omega)$ и свободной $I_{\mathrm{SLG}}^{0}(\omega)$ графеновых структур. Все энергетические величины измеряются в единицах $E_{g} / 2: \xi=4$, $\bar{\Gamma}_{s c} / \pi=0.1$. Изображены только правые половины симметричных функций.

$(\xi, \infty)$. Тогда для нормировочного множителя получаем $L^{-1}=\left(E_{g}^{2} / 4 \xi^{2}\right)\left(k_{1}-k_{2}\right)+k_{2}$, так что

$$
C_{Q}^{\mathrm{ESLG}}=\left(2 e^{2} T L / S \xi^{2}\right)\left[k_{1} B\left(e_{g} / 2, v^{*}\right)+k_{2} I\left(e_{g} / 2, \bar{\xi} ; v^{*}\right)\right] .
$$

Вводя $\Delta C_{Q}^{\mathrm{ESLG}}=C_{Q}^{\mathrm{ESLG}}-C_{Q}^{\mathrm{SLG}}$ и учитывая, что в низкоэнергетическом приближении $C_{Q}^{\mathrm{SLG}}=\left(2 e^{2} T / S \xi^{2}\right)$ $\times B\left(\bar{\xi} ; v^{*}\right)[19]$, получим

$$
\begin{aligned}
\Delta C_{Q}^{\mathrm{ESLG}}= & \left(2 e^{2} T / S \xi^{2}\right)\left[L\left(k_{1}-k_{2}\right) B\left(e_{g} / 2, v^{*}\right)\right. \\
& \left.+\left(L k_{2}-1\right) B\left(\bar{\xi} ; v^{*}\right)\right]
\end{aligned}
$$

В первом порядке по $\bar{\Gamma}_{s c} / E_{g}$ имеем $L \approx 1-\bar{\Gamma}_{s c} E_{g}^{3} / \pi \xi^{4}$, так что получим

$$
\begin{aligned}
\Delta C_{Q}^{\mathrm{ESLG}} \approx & \frac{8 e^{2} T \bar{\Gamma}_{s c}}{\pi S \xi^{2} E_{g}} F_{\mathrm{ESLG}}, \\
F_{\mathrm{ESLG}} \approx & \left(1+\frac{E_{g}^{2}}{4 \xi^{2}}\right) B\left(e_{g} / 2, v^{*}\right) \\
& -\frac{E_{g}^{2}}{4 \xi^{2}}\left(1+\frac{E_{g}^{2}}{\xi^{2}}\right) B\left(\bar{\xi}, v^{*}\right) .
\end{aligned}
$$

При комнатной температуре $e_{g} / 2 \gg 1$ и $v^{*} \ll e_{g} / 2$, имеем

$$
F_{\mathrm{ESLG}} \approx\left(1-\frac{E_{g}^{4}}{4 \xi^{4}}\right) \ln \left\{2\left[1+\operatorname{ch}\left(2 v^{*}\right)\right]\right\},
$$


так что $\Delta C_{Q}^{\mathrm{ESLG}}>0$ и увеличивается с ростом $v^{*}$. При этом

$$
\eta_{\mathrm{ESLG}}=\frac{\Delta C_{Q}^{\mathrm{ESLG}}}{C_{Q}^{\mathrm{SLG}}}=\frac{4 \bar{\Gamma}_{s c}}{\pi E_{g}}\left(1-\frac{E_{g}^{4}}{4 \xi^{4}}\right) .
$$

3.2.3. Графеноподобные соединения. Гетероконтакты GLC - полупроводниковая подложка рассматривались в работе [37]. В зависимости от взаиморасположения и ширины щели GLC $2 \Delta$ и запрещенной зоны полупроводника $E_{g}$ возникают различные типы гетеропереходов (см. рис. 1 в [37]). Здесь в качестве иллюстрации мы рассмотрим простейшую ситуацию, когда центры щели и запрещенной зоны совпадают и приняты за нуль энергии. При этом возможны два случая: $2 \Delta>E_{g}$ и $2 \Delta<E_{g}$ (см. порядковые оценки $\Delta$ в [29]). Первый случай реализуется для полупроводниковых подложек $\left(\mathrm{Si}, \mathrm{SiC}, \mathrm{A}^{\mathrm{III}} \mathrm{B}^{\mathrm{V}}[38,39]\right)$, второй - для диэлектрических подложек (h-BN, $\mathrm{AlN}, \mathrm{SiO}_{2}$ [40-43]). Это так называемые охватывающие (straddling) гетеропереходы [38]. В дальнейшем будем рассматривать плоские эпитаксиальные слои, используя низкоэнергетическое приближение и предел слабой связи $\bar{\Gamma}_{s c} \ll \min \left\{\Delta, E_{g} / 2\right\}$. Для случая $2 \Delta>E_{g}$ приведенная плотность состояний $I_{\mathrm{EGLC}}^{*}(\omega)=2 \hat{I}(\omega) / E_{g}=\rho_{\mathrm{EGLC}}(\omega) \xi^{2} / E_{g}$ представлена на pис. 5 , для случая $2 \Delta<E_{g}-$ на рис. 6 . Там же для сравнения изображена приведенная плотность состояний свободного GLC $I_{\mathrm{GLC}}^{0}(\omega)=\rho_{\mathrm{GLC}}(\omega) \xi^{2} / E_{g}$. Отметим, что малые пики $\rho_{\mathrm{EGLC}}(\omega)$ (рис. 5 и 6 ) связаны со ступенчатыми плотностями состояний подложки и GLC.

Начнем со случая $2 \Delta>E_{g}$ (рис. 5). Аппроксимируя интеграл $\hat{I}(\omega)$ (приведенная плотность состояний) линейной функцией $\hat{k} \omega$, при $\bar{\Gamma}_{s c} \neq 0$ получим

$$
C_{Q}^{\mathrm{EGLC}} \approx\left(2 e^{2} T M \hat{k} / \xi^{2} S\right) I\left(e_{g} / 2, x_{\max } ; v^{*}\right),
$$

где $\hat{k}=1-4 \bar{\Gamma}_{s c} E_{g} / \pi\left(4 \Delta^{2}-E_{g}^{2}\right), M-$ нормировочный множитель. Легко показать, что $M \hat{k}=$ $=\left[1+\left(4 \Delta^{2}-E_{g}^{2}\right) / 4 \xi^{2}\right]^{-1}$. При $\bar{\Gamma}_{s c}=0$ выражение (26) переходит в $\quad C_{Q}^{\mathrm{GLC}} \approx\left(2 e^{2} T / \xi^{2} S\right) I\left(\delta, x_{\max } ; v^{*}\right)$. Тогда разность $\Delta C_{Q}^{\mathrm{EGLC}}=C_{Q}^{\mathrm{EGLC}}-C_{Q}^{\mathrm{GLC}}$ имеет вид

$$
\begin{gathered}
\Delta C_{Q}^{\mathrm{EGLC}} \approx\left(2 e^{2} T / \xi^{2} S\right) F_{\mathrm{EGLC}}, \\
F_{\mathrm{EGLC}}=M \hat{k} I\left(e_{g} / 2, x_{\max } ; v^{*}\right)-I\left(\delta, x_{\max } ; v^{*}\right) .
\end{gathered}
$$

При $v^{*} \ll e_{g} / 2$ получаем $F_{\mathrm{EGLC}} \approx 2 \operatorname{ch}\left(2 v^{*}\right) e_{g} \exp \left(-e_{g}\right) \ll 1$ и

$$
\eta_{\mathrm{EGLC}} \approx \frac{F_{\mathrm{EGLC}}}{\left[1+\left(4 \Delta^{2}-E_{g}^{2}\right) / 4 \xi^{2}\right] \ln \left\{2\left[1+\operatorname{ch}\left(2 v^{*}\right)\right]\right\}} .
$$

Таким образом, $\Delta C_{Q}^{\mathrm{ESLG}}>0$ и растет с ростом $v^{*}$, но $F_{\mathrm{EGLC}}$ и $\eta_{\mathrm{EGLC}}-$ экспоненциально малые величины.

Усложним модель. Приведенная плотность состояний EGLC, представленная на рис. 5, может быть задана в виде

$$
\hat{I}(\omega) \approx \begin{cases}0, & |\omega| \leq E_{g} / 2, \\ \bar{\Gamma}_{s c} b / \pi, & E_{g} / 2<|\omega| \leq \Delta, \\ \hat{k}|\omega|, & \Delta<|\omega| \leq x_{\max }\end{cases}
$$

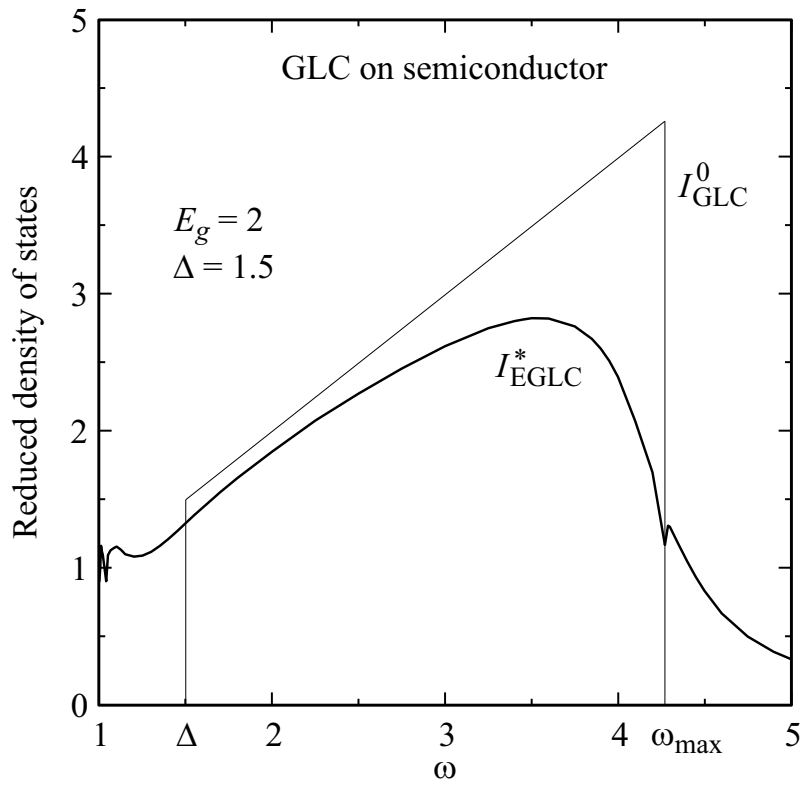

Рис. 5. Графеноподобное соединение (GLC) на полупроводнике: приведенная плотность состояний $I_{\mathrm{ESLG}}^{*}(\omega)$. Изображена также приведенная плотность состояний свободного GLC $I_{\mathrm{GLC}}^{0}(\omega)$ при $\varepsilon_{\mathrm{D}}$. Все энергетические величины измеряются в единицах $E_{g} / 2: \Delta=1.5, \xi=4, \bar{\Gamma}_{s c} / \pi=0.1$. Изображены только правые половины симметричных функций.

где $b-$ безразмерная константа, откуда получаем

$$
C_{Q}^{\mathrm{EGLC}} \approx\left(2 e^{2} T M^{\prime} / \xi^{2} S\right)\left(\bar{J}_{1}+\bar{J}_{2}\right),
$$

где $M^{\prime}=\left[\hat{k}+b \bar{\Gamma}_{s c}\left(2 \Delta-E_{g}\right) / \pi \xi^{2}\right]^{-1}, \bar{J}_{1}=\hat{k} I\left(\delta, x_{\max } ; v^{*}\right)$, $\bar{J}_{2}=\left(b \gamma_{s c} / \pi\right)\left[C\left(\delta, v^{*}\right)-C\left(e_{g} / 2, v^{*}\right)\right], \gamma_{s c}=\bar{\Gamma}_{s c} / 2 T$. Toгда

$$
\begin{gathered}
\Delta C_{Q}^{\mathrm{EGLC}} \approx\left(2 e^{2} T / \xi^{2} S\right) F_{\mathrm{EGLC}}^{\prime}, \\
F_{\mathrm{EGLC}}^{\prime}=(M \hat{k}-1) I\left(\delta, x_{\max } ; v^{*}\right)+M \bar{J}_{2} .
\end{gathered}
$$

Так как $M \approx 1+4 \bar{\Gamma}_{s c} E_{g} / \pi\left(4 \Delta^{2}-E_{g}^{2}\right)-b \bar{\Gamma}_{s c}\left(2 \Delta-E_{g}\right) / \pi \xi^{2}$, в первом порядке по $\bar{\Gamma}_{s c}$ функция $F_{\mathrm{EGLC}}^{\prime} \approx$ $\approx-\left[b \bar{\Gamma}_{s c}\left(2 \Delta-E_{g}\right) / \pi \xi^{2}\right] I\left(\delta, x_{\max } ; v^{*}\right)+M \bar{J}_{2}$. При $v^{*} \ll e_{g} / 2$ получим

$$
F_{\mathrm{EGLC}}^{\prime} \approx\left(4 b \gamma_{s c} / \pi\right) \operatorname{ch}\left(2 v^{*}\right) \exp \left(-e_{g}\right)
$$

Таким образом, в рамках первой и второй моделей получаем соответственно

$$
\begin{gathered}
\left(\Delta C_{Q}^{\mathrm{EGLC}}\right)_{1,2} \approx \bar{C}_{1,2} \operatorname{ch}(V / T) \exp \left(-E_{g} / 2 T\right), \\
\bar{C}_{1}=2 e^{2} E_{g} / S \xi^{2}, \quad \bar{C}_{2}=8 b e^{2} \bar{\Gamma}_{s c} / \pi S \xi^{2} .
\end{gathered}
$$

Итак, с качественной точки зрения обе модели приводят к одинаковым результатам.

Теперь перейдем к случаю $2 \Delta<E_{g}$. Сравнение рис. 6 и 4 показывает, что в данном случае можно воспользоваться тем же приемом, что и при оценке KE для SLG 


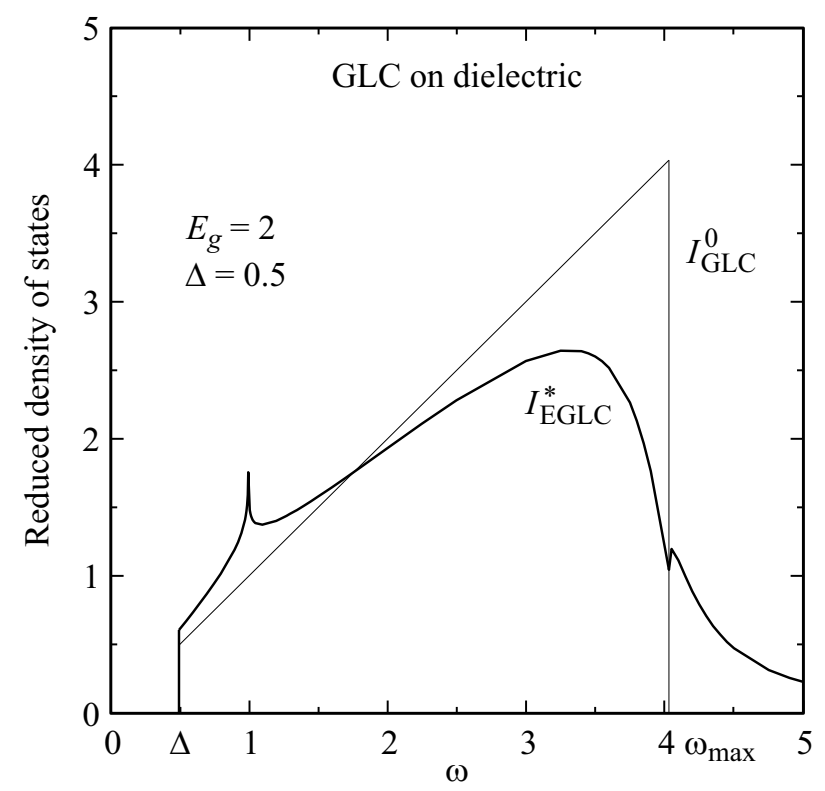

Рис. 6. Графеноподобное соединение (GLC) на диэлектрике: то же, что на рис. 5 , но для $\Delta=0.5$.

на полупроводнике (диэлектрике). Тогда запишем

$$
\begin{aligned}
C_{Q i}^{\mathrm{EGLC}} & =\left(2 e^{2} T N / S \xi^{2}\right)\left(\widetilde{J}_{1}+\widetilde{J}_{2}\right), \\
\widetilde{J}_{1,2} & =\widetilde{k}_{1,2} \int_{\widetilde{a}_{1,2}}^{\widetilde{b}_{1,2}} x A\left(x, v^{*}\right) d x,
\end{aligned}
$$

где $\quad \widetilde{a}_{1}=\delta, \quad \widetilde{b}_{1}=\widetilde{a}_{2}=e_{g} / 2, \quad \widetilde{b}_{2}=x_{\max }, \quad N=$ $=\left(\left[\left(E_{g}^{2}-4 \Delta^{2}\right) / 4 \xi^{4}\right]\left(\widetilde{k}_{1}-\widetilde{k}_{2}\right)+k_{2}\right)^{-1}-$ нормировочный множитель. В интервале $\left(\Delta, E_{g} / 2\right)$ аппроксимируем плотность состояний $\rho_{\mathrm{EGLC}}(\omega) \propto \omega\left[1-\left(\partial \Lambda_{s c}(\omega) / \partial \omega\right)_{\omega=\Delta}\right]$ $=\widetilde{k}_{1} \omega$, где $\widetilde{k}_{1} \approx 1+4 \bar{\Gamma}_{s c} E_{g} / \pi\left(E_{g}^{2}-4 \Delta^{2}\right)$. Аналогичным образом для интервала $\left(E_{g} / 2, \omega_{\max }\right)$ получим $\widetilde{k}_{2} \approx 1-4 \bar{\Gamma}_{s c} E_{g} / \pi\left(4 \omega_{\max }^{2}-E_{g}^{2}\right)$. Учитывая, что при этом $N=1$, запишем

$$
\begin{gathered}
\Delta C_{Q i}^{\mathrm{EGLC}}=\frac{2 e^{2} T E_{g} \bar{\Gamma}_{s c}}{\pi S \xi^{4}} F_{\mathrm{EGLC}}^{\prime \prime}, \\
F_{\mathrm{EGLC}}^{\prime \prime}=\xi^{2}\left(\frac{I\left(\delta, e_{g} / 2 ; v^{*}\right)}{E_{g}^{2}-4 \Delta^{2}}+\frac{I\left(e_{g} / 2, x_{\max } ; v^{*}\right)}{\omega_{\max }^{2}-E_{g}^{2}}\right) .
\end{gathered}
$$

При $v^{*} \ll \delta$ имеем

$$
F_{\mathrm{EGLC}}^{\prime \prime} \approx 4\left[\xi^{2} /\left(E_{g}^{2}-4 \Delta^{2}\right)\right] \operatorname{ch}\left(2 v^{*}\right) \delta \exp (-2 \delta)
$$

и $\quad \eta_{\mathrm{EGLC}} \approx\left[8 E_{g} \bar{\Gamma}_{s c} / \pi\left(E_{g}^{2}-\Delta^{2}\right)\right] \operatorname{ch}\left(2 v^{*}\right) \delta \exp (-2 \delta) \ll 1$. При $\delta \ll v^{*} \ll e_{g} / 2$ получим

$$
F_{\mathrm{EGLC}}^{\prime \prime} \approx 4\left[\xi^{2} /\left(E_{g}^{2}-4 \Delta^{2}\right) \ln \left\{2\left[1+\operatorname{ch}\left(2 v^{*}\right)\right]\right\}\right.
$$

и $\eta_{\mathrm{EGLC}} \approx\left[2 E_{g} \bar{\Gamma}_{s c} / \pi\left(E_{g}^{2}-\Delta^{2}\right)\right] \ln \left\{2\left[1+\operatorname{ch}\left(2 v^{*}\right)\right]\right\}$. Отсюда следует, что $\Delta C_{Q}^{\mathrm{EGLC}}>0$, т.е. KE EGLC выше, чем в случае свободного GLC.
Таким образом, для всех рассмотренных эпитаксиальных структур взаимодействие с подложкой приводит к увеличению КЕ, пропорциональному во всех случаях $V^{*}$, a также $\Gamma_{m}$ в случае металлической подложки и $\bar{\Gamma}_{s c}$ в случае диэлектрической подложки. При $2 \Delta>E_{g}$ явная зависимость КЕ от $\bar{\Gamma}_{s c}$ может отсутствовать, но это следствие принятого модельного описания. Отметим также, что приращение КЕ равно нулю при $\Gamma_{m}=0$ и $\bar{\Gamma}_{s c}=0$, но остается конечным при $V^{*}=0$.

\section{4. Заключение}

Итак, в настоящей работе мы использовали простые модели плотности состояний аморфного графена, $\rho_{\mathrm{ASLG}}(\omega)$, и аморфного графеноподобного соединения, $\rho_{\text {AGLC }}(\omega)$. По сравнению с кристаллической структурой в первом случае имеет место увеличение плотности состояний в области малых энергий (рис. 1), во втором появление конечной плотности состояний в области щели (рис. 2). Оценки показали уменьшение значений КЕ при аморфизации. Для описания плотностей состояний эпитаксиальных слоев SLG и GLC, сформированных на металлической и полупроводниковой (диэлектрической) подложках, использовалось низкоэнергетическое приближение и приближение слабой связи слой-подложка. Оценки показали уменьшение КЕ в случае металлической подложки и увеличение КЕ в случае полупроводниковой (диэлектрической) подложки.

При оценках КЕ мы прибегали к ряду аппроксимаций. Поэтому нелишними представляются следующие пояснения. В низкоэнергетическом приближении электронный спектр графена имеет вид $\varepsilon_{ \pm}(\mathbf{q})= \pm \hbar v_{\mathrm{F}}|\mathbf{q}|$, где $v_{\mathrm{F}}=3 a t / 2 \hbar-$ скорость Ферми, $a-$ расстояние между ближайшими соседями, $\mathbf{q}-$ волновой вектор, отсчитываемый от точки Дирака, $\hbar-$ приведенная постоянная Планка, знаки „士“ относятся соответственно к валентной зоне и зоне проводимости. При этом плотность состояний имеет вид $\rho_{\mathrm{SLG}}(\omega)=4 S|\omega| / \hbar^{2} v_{\mathrm{F}}^{2}[28]$. Такая форма записи удобна для описания дифференциальных характеристик спектра вблизи точки Дирака. Если, однако, требуется найти интегральную характеристику - например, число заполнения атома углерода недопированного графена $n_{\mathrm{SLG}}=\int_{-\infty}^{0} \rho_{\mathrm{SLG}}(\omega) d \omega=1$, то приходится вводить энергию обрезания $\xi$ и, оставаясь в пределах низкоэнергетического приближения, записывать плотность состояний в виде $\rho_{\mathrm{SLG}}(\omega)=2|\omega| / \xi^{2}$. Далее, в модельном представлении плотности состояний кристаллического графена [44], на основе которого предложено выражение (1), игнорируются особенности Ван Хова. При дифференцировании модельной плотности состояний в окрестности $\omega= \pm t$ получим конечные, а не бесконечные значения производных. Таким образом, при интегрировании неточности модельного представления сглаживаются и становятся приемлемыми. 
Автору хотелось бы надеяться, что полученные в настоящей работе результаты послужат стимулом для соответствующих экспериментальных исследований.

\section{Приложение}

Определим функции

$$
B\left(y, v^{*}\right)=\int_{0}^{y} x A\left(x, v^{*}\right) d x
$$

и

$$
I\left(y_{1}, y_{2} ; v^{*}\right)=\int_{y_{1}}^{y_{2}} x A\left(x, v^{*}\right) d x=B\left(y_{2}, v^{*}\right)-B\left(y_{1}, v^{*}\right),
$$

где $A\left(x, v^{*}\right)=\operatorname{sch}^{2}\left(x-v^{*}\right)+\operatorname{sch}^{2}\left(x+v^{*}\right)$ и $v^{*}-$ безразмерный параметр. Легко показать [45], что

$$
\begin{gathered}
B\left(y, v^{*}\right)=y C\left(y, v^{*}\right)-\ln \frac{\operatorname{ch}\left(y-v^{*}\right) \cdot \operatorname{ch}\left(y+v^{*}\right)}{\operatorname{ch}^{2} v^{*}} \\
C\left(y, v^{*}\right)=\operatorname{th}\left(y-v^{*}\right)+\operatorname{th}\left(y+v^{*}\right)
\end{gathered}
$$

В дальнейшем предполагается, что $y \gg 1$. При $y \gg v^{*} \mathrm{c}$ точностью до ехр $(-2 y)$ получим

$$
\begin{gathered}
C\left(y, v^{*}\right) \approx-4 \exp (-2 y) \operatorname{ch}\left(2 v^{*}\right), \\
B\left(y, v^{*}\right) \approx \ln \left\{2\left[1+\operatorname{ch}\left(2 v^{*}\right)\right]\right\}-4 y \exp (-2 y) \operatorname{ch}\left(2 v^{*}\right) \\
-2 \exp (-2 y) \operatorname{ch}\left(2 v^{*}\right) .
\end{gathered}
$$

При $v^{*} \gg y$ с точностью до $\exp \left(-2 v^{*}\right)$ имеем

$$
\begin{gathered}
C\left(y, v^{*}\right) \approx 4 \exp \left(-2 v^{*}\right) \operatorname{sh}(2 y), \\
B\left(y, v^{*}\right) \approx \ln \left\{2\left[1+\operatorname{ch}\left(2 v^{*}\right)\right]\right\}+4 y \exp \left(-2 v^{*}\right) \operatorname{sh}(2 y) \\
-2 \exp \left(-2 v^{*}\right) \operatorname{ch}(2 y) .
\end{gathered}
$$

Если $y_{2}>y_{1} \gg v^{*}$, то с точностью до $y_{1,2} \exp \left(-2 y_{1,2}\right)$ получаем

$$
I\left(y_{1}, y_{2} ; v^{*}\right) \approx 4 \operatorname{ch}\left(2 v^{*}\right)\left[y_{1} \exp \left(-2 y_{1}\right)-y_{2} \exp \left(-2 y_{2}\right)\right]
$$

если $y_{2} \gg v^{*} \gg y_{1}$, то с той же точностью имеем

$$
\begin{aligned}
I\left(y_{1}, y_{2} ; v^{*}\right) \approx & \ln \left\{2\left[1+\operatorname{ch}\left(2 v^{*}\right)\right]\right\}+4 \operatorname{ch}\left(2 v^{*}\right) \\
& \times\left[y_{1} \exp \left(-2 y_{1}\right)-y_{2} \exp \left(-2 y_{2}\right)\right]
\end{aligned}
$$

если $v^{*} \gg y_{2} \gg y_{1}$, то с точностью до $y_{1,2} \operatorname{sh}\left(2 y_{1,2}\right)$ находим

$$
I\left(y_{1}, y_{2} ; v^{*}\right) \approx 4 \exp \left(-2 v^{*}\right)\left[y_{2} \operatorname{sh}\left(2 y_{2}\right)-y_{1} \operatorname{sh}\left(2 y_{1}\right)\right] .
$$

\section{Конфликт интересов}

Автор заявляет об отсутствии конфликта интересов.

\section{Список литературы}

[1] S. Luryi. Appl. Phys. Lett., 96, 501 (1988).

[2] D.L. John, L.C. Castro, D.L. Pulfrey. J. Appl. Phys., 96, 5180 (2004).

[3] T. Fang, A. Konar, H. Xing, D. Jena. Appl. Phys. Lett., 91, 092109 (2007)

[4] J. Guo, Y. Yoon, Y. Ouyang. Nano Lett., 7, 1935 (2007).

[5] G.S. Kliros. Romanian J. Inform. Sci. Technol., 13. 332 (2010).

[6] M.V. Cheremisin. Physica E, 69, 153 (2015).

[7] Z.Z. Alisultanov, M.S. Reis. EPL, 113, 28004 (2016).

[8] A.B.G. Trabelsi, F.V. Kusmartsev, D.M. Forrester, O.E. Kusmartseva, M.B. Gaifullin, P. Cropper, M. Oueslati. J. Mater. Chem. C, 4, 5829 (2016).

[9] С.Ю. Давыдов, А.А. Лебедев, П.В. Булат, А.В. Зубов. Письма ЖТФ, 46 (15), 7 (2020).

[10] F. Su, L. Huo, Q. Kong, L. Xie, C. Chen. Catalysts, 8, 444 (2018).

[11] Q. Xu, G. Yang, X. Fan, W. Zheng. ASC Omega, 4, 13209 (2019).

[12] T. Sruthi, K. Tarafder. Bull. Mater. Sci., 42, 257 (2019). https://doi.org/10.1007/s12034-019-1952-8

[13] Y. Shao, M.F. El-Kady, L.J. Wang, Q. Zhang, Y. Li, H. Wang, M.F. Mousaviae, R.B. Kaner. Chem. Soc. Rev., 44, 3639 (2015).

[14] M.F. El-Kady, Y. Shao, R.B. Kaner. Nature Rev. Mater., 1, 16033 (2016).

[15] П.В. Ратников, А.П. Силин. УФН, 188, 1294 (2018).

[16] C.-J. Tong, H. Zhang, Y.-N. Zhang, H. Liu, L.-M. Liu. J. Mater Chem. A, 2, 17971 (2014).

[17] A.K. Singh, H.L. Zhuang, R.G. Hennig. Phys. Rev. B, 89, 245431 (2014).

[18] D. Kecik, A. Onen, M. Konuk, E. Gürbüz, F. Ersan, S. Cahangirov, E. Aktürk, E. Durgun, S. Ciraci. Appl. Phys. Rev., 5, 011105 (2018)

[19] С.Ю. Давыдов, А.А. Лебедев, А.В. Зубов, П.В. Булат. Письма ЖТФ, 46 (23), 19 (2020).

[20] V. Kapko, D.A. Darbold, M.F. Thorpe. Phys. Status Solidi B, 247, 1197 (2010).

[21] M. Zheng, K. Takei, B. Hsia, H. Fang, X. Zhang, N. Ferralis, H. Ko, Y.-L. Chuen, Y. Zang, R. Maboudian, A. Javey. Appl. Phys. Lett., 96, 063110 (2010).

[22] J. Kotakoski, A.V. Krasheninnikov, U. Kaizer, J.C. Meyer. Phys. Rev. Lett., 106, 105505 (2011).

[23] D.V. Tuan, A. Kumar, S. Roche, F. Ortmann, M.F. Thorpe, P. Ordejon. Phys. Rev. B, 86, 121408 (R) (2012).

[24] С.Ю. Давыдов. ФТП, 50, 382 (2016).

[25] Дж. Займан. Модели беспорядка (М., Мир, 1982).

[26] С.Ю. Давыдов. ФТТ, 57, 1017 (2015).

[27] N.M.R. Peres, F. Guinea, A.H. Castro Neto. Phys. Rev. B, 73, 125411 (2006).

[28] A.H. Castro Neto, F. Guinea, N.M.R. Peres, K.S. Novoselov, A.K. Geim. Rev. Mod. Phys., 81, 109 (2009).

[29] С.Ю. Давыдов. ФТТ, 58, 779 (2016).

[30] С.Ю. Давыдов, А.А. Лебедев, О.В. Посредник. Элементарное введение в теорию наносистем (СПб., Изд-во „Лань“, 2014) гл. 8.

[31] Ч. Киттель. Квантовая теория твердых тел (М., Наука, 1967) гл. 18.

[32] С.Ю. Давыдов. ФТП, 47, 97 (2013).

[33] С.Ю. Давыдов. ФТП, 45, 1102 (2011).

[34] С.Ю. Давыдов. Письма ЖТФ, 39 (2), 7 (2013). 
[35] С.Ю. Давыдов. ЖТФ, 84 (4), 155 (2014).

[36] S. Mammadov, J. Ristein, J. Krone, C. Raidel, M. Vanke, V. Weismann, F. Speck, T. Seyller. 2D Mater., 4, 015043 (2017).

[37] С.Ю. Давыдов. ФТТ, 58, 1182 (2016).

[38] Ф. Бехштедт, Р. Эндерлайн. Поверхности и границы раздела полупроводников (М., Мир, 1990).

[39] I. Vurgaftmana, J.R. Meyer, L.R. Ram-Mohan. Appl. Phys. Rev., 89, 5815 (2001).

[40] M.E. Levinshtein, S.L. Rumyantsev, M.S. Shur. Properties of Advanced Semiconductor Materials (N. Y., Wiley, 2001).

[41] D. Wickramaratne, L. Weston, C.G. Van de Walle. J. Phys. Chem. C, 122, 25524 (2018).

[42] E. Vella, F. Messina, M. Cannas, R. Boscaino. Phys. Rev. B, 83, 174201 (2011).

[43] С.С. Некрашевич, В.А. Гриценко. ФТТ, 56, 209 (2014).

[44] С.Ю. Давыдов, Г.И. Сабирова. ФТТ, 53, 608 (2011).

[45] Г.Б. Двайт. Таблицы интегралов и другие математические формуль (СПб., Изд-во „Лань“, 2009).

Редактор Л.В. Шаронова

\section{Model estimates of quantum capacitance for amorphous and epitaxial graphene-like compounds}

S.Yu. Davydov

loffe Institute, 194021 St. Petersburg, Russia

Abstract Models for the densities of states of amorphous graphene and binary graphene-like compounds (GLC), which are used for the estimates of the quantum capacitance, are proposed. It is shown that the transition from crystalline to amorphous state decreases quantum capacitance. The same effect is realized when graphene and GLC couple with a metallic substrate, while in the case of a semiconductor (dielectric) substrate the increase of quantum capacitance takens place. 